\title{
ANÁLISE PROBABILÍSTICA DA VELOCIDADE MÉDIA E CARACTERIZAÇÃO DA DIREÇÃO PREDOMINANTE DO VENTO NO MUNICÍPIO DA LAPA/PR
}

\section{Probabilistic analysis of the average speed and characterization of the predominant wind direction in the city of Lapa/PR}

\author{
Gustavo Castilho BERUSKI ${ }^{1}$ \\ Maysa de Lima LEITE² \\ Jorim Sousa das VIRGENS FILHO ${ }^{3}$ \\ Patrícia Alves ADACHESKI ${ }^{4}$ \\ André Vicente de OLIVEIRA ${ }^{5}$
}

\begin{abstract}
RESUMO
Com o alto nível de poluentes emitidos no ambiente pela queima de combustíveis fósseis, tem-se procurado fontes alternativas de geração de energia, como a eólica. A partir de uma série de 19 anos de dados diários de velocidade média e direção predominante dos ventos da Lapa, PR, caracterizou-se o comportamento do vento na região por meio de distribuições probabilísticas. Dentre estas, a que melhor se ajustou aos dados de velocidade média foi a distribuição Gama. A direção predominante foi a Leste, seguida de Nordeste e Norte. Verificou-se que a região estudada sofre influência de diversos fatores climáticos.
\end{abstract}

Palavras-chave: Velocidade média do vento; Direção predominante; Distribuições de probabilidade.

\begin{abstract}
Due to the high level of pollutants emitted in the environment by the fossil fuel burning, alternative sources of energy generation have been searched, as the aeolian one. From a series of 19 years of daily data of average speed and the predominant direction of the wind in the city of Lapa, PR, this study characterized the behavior of the wind in the region by using probability distributions. Among these, the one that best adjusted to the data of average speed of wind was the Gamma distribution. The predominant direction was the Eastern one, followed by Northeastern and Northern one. It was noticed that the studied region suffers influence from diversified climatic factors.
\end{abstract}

Keywords: Average speed of the wind; Predominant direction; Probability distributions. 


\section{INTRODUÇÃO}

Atualmente obtem-se energia através da queima de combustíveis fósseis. Porém sabe-se hoje que a queima de tais combustíveis gera gases $\mathrm{CO}_{2}$ e $\mathrm{CO}$, que são nocivos tanto para o homem quanto para o meio ambiente. Estes gases atuam como promotores de efeitos como o aquecimento global e o efeito estufa (SILVA; BURGUEÑO, 2001).

Em virtude desses fatos, tem-se procurado fontes alternativas para a obtenção de energia, como é o caso das energias solar e eólica, que são fontes renováveis, limpas e que não poluem o meio ambiente (OLIVEIRA et al., 2003). Entretanto, para que se obtenham resultados satisfatórios com relação à energia eólica, é necessário efetuar estudos prévios da região, analisando-se características eólicas como velocidade e direção predominante do vento, determinando se tal localidade possui potencial eólico favorável.

A direção dos ventos é uma das características cujo estudo é de fundamental importância, pois a partir desta pode-se determinar locais de instalação de distritos industriais, quebra-ventos, estábulos e torres de captação de energia (DA SILVA et al., 1997). Do ponto de vista agronômico, a direção dos ventos pode atuar no posicionamento de culturas nas lavouras e está também diretamente ligada ao fenômeno de polinização (MUNHOZ; GARCIA, 2008).

Além da direção predominante, a velocidade média dos ventos também é de grande interesse, pois trata-se de uma informação fundamental para verificar se uma determinada região possui um potencial eólico satisfatório, viabilizando tecnicamente a instalação de torres de captação de energia.

Para se estimar o comportamento dos ventos em determinada região, utiliza-se o tratamento estatístico dos dados obtidos (ALDABÓ, 2002), cujas técnicas têm sido amplamente aplicadas no Brasil e em vários outros países.

O desenvolvimento deste trabalho teve como objetivo verificar dentre as distribuições probabilísticas Normal, Beta, Gama, Weibull e Rayleigh qual apresenta o melhor ajuste mensal aos dados diários de velocidade média dos ventos para a localidade da Lapa, no Estado do Paraná, além de determinar a direção predominante do vento neste município.

\section{METODOLOGIA}

Os dados analisados foram cedidos pelo Instituto Agronômico do Paraná (IAPAR) e obtidos junto a uma Estação Meteorológica localizada no município da Lapa (latitude $25^{\circ} 47^{\prime} \mathrm{S}$, longitude $49^{\circ} 46^{\prime} \mathrm{W}$ e altitude de $910 \mathrm{~m}$ ). Estes foram coletados por meio de um Anemógrafo Universal, da marca R. Fuess, com seus sensores localizados a uma altura de $10 \mathrm{~m}$ acima do nível do solo. Os dados consistiam de registros diários de velocidade média e direção predominante dos ventos, sendo estes de uma série de 19 anos de registros, coletados no período de 1988 a 2007.

Após a obtenção dos dados, efetuou-se a triagem dos mesmos, em planilhas do Microsoft Excel 2003 e em seguida foi realizada a sua estatística descritiva de acordo com Assis et al. (1996). Em seguida, aplicaramse as distribuições probabilísticas Normal, Beta, Gama, Weibull e Rayleigh, descritas detalhadamente abaixo.

A distribuição Normal é expressa pela seguinte função de densidade de probabilidade:

$$
f(v)=\frac{1}{\sigma \sqrt{2 \pi}} e^{-\frac{(v-\mu)^{2}}{2 \sigma^{2}}}
$$

onde $\mu$ representa a média aritmética e $\sigma$ o desviopadrão, cujas estimativas de máxima verossimilhança são dadas respectivamente por $\bar{v}$ e s, que são obtidas por:

$$
\begin{aligned}
& \bar{v}=\frac{\sum_{i=1}^{N} v_{i}}{N} \\
& S=\sqrt{\frac{\sum_{i=1}^{N}\left(v_{i}-\bar{v}\right)^{2}}{N-1}}
\end{aligned}
$$

Sua função cumulativa de probabilidade é dada por:

$$
F(v)=\frac{1}{\sigma \sqrt{2 \pi}} \int_{-\infty}^{v} e^{\frac{(v-\mu)^{2}}{2 \sigma^{2}}} d v
$$


A função de densidade de probabilidade pode ser expressa da seguinte forma (FALLS, 1973):

$$
\mathrm{F}(\mathrm{v})=\frac{1}{(b-a)} \frac{\Gamma(p+q)}{\Gamma(p) \Gamma(q)}\left(\frac{v-a}{b-a}\right)^{p-1}\left(1-\frac{v-a}{b-a}\right)^{q-1}
$$

onde $a$ e $b$ correspondem ao menor e maior valor da série de dados, respectivamente, $\Gamma$ é o símbolo da função Gama das respectivas variáveis, $p$ e $q$ são parâmetros da distribuição Beta e $v$ é um valor qualquer da variável em análise. A estimativa dos parâmetros $p e$ $q$ pode ser realizada a partir do método dos momentos (PEARSON, 1934).

$$
\begin{aligned}
& p=\frac{\mu_{1}\left(\mu_{1}-\mu_{2}\right)}{\left[\mu_{2}-\left(\mu_{1}\right)^{2}\right]} \\
& q=\frac{\left(1-\mu_{1}\right)\left(\mu_{1}-\mu_{2}\right)}{\left[\mu_{2}-\left(\mu_{1}\right)^{2}\right]}
\end{aligned}
$$

onde, $\mu_{1}$ corresponde ao momento de ordem 1 para a variável $v$ e $\mu_{2}$ ao momento de ordem 2 para a variável $\mathrm{v}$, dentro de uma série de $\mathrm{N}$ dados. Estes termos podem ser estimados a partir da seguinte análise:

$$
\begin{aligned}
& \mu_{1}=\frac{\sum_{i=1}^{N} v_{i}}{N} \\
& \mu_{2}=\frac{\sum_{i=1}^{N} v_{i}^{2}}{N}
\end{aligned}
$$

Para a estimativa dos valores de ocorrência de probabilidade, através da distribuição Beta, a equação (5) deve ser adimensionalizada para um intervalo compreendido entre [0 e 1]. A variável adimensionalizada v' toma então a seguinte forma:

$$
v^{\prime}=\frac{v-a}{b-a}
$$

sugerindo que a função de densidade de probabilidade Beta assuma a seguinte forma:

$$
f\left(v^{\prime}\right)=\frac{\Gamma(p+q)}{\Gamma(p) \Gamma(q)}\left(v^{\prime}\right)^{p-1}\left(1-v^{\prime}\right)^{\mathrm{q}-1}
$$

onde $0 \leq v^{\prime} \geq 1$, para $p>1$ e $q>1$.

A integração numérica da equação (9) confere os valores da probabilidade de ocorrência para um valor de $\checkmark$ qualquer dentro do intervalo considerado.

A função de densidade de probabilidade Gama é expressa por:

$$
f(v)=\frac{v^{\alpha-1} e^{-v / \beta}}{\beta^{\alpha} \Gamma(\alpha)}
$$

onde: $\alpha$ = Parâmetro de forma, estimado pelo método de Greenwood ; Durand (1960), dado por:

$$
\alpha=\frac{8,898919+9,05995 y+0.9775373 y^{2}}{y\left(17,79728+11,968477 y+y^{2}\right)}
$$

$$
\text { Para } 0 \leq y \leq 0,5772
$$

ou

$$
\alpha=\frac{0,5000876+0,1648852 \mathrm{y}-0,0544274 \mathrm{y}^{2}}{\mathrm{y}}
$$

$$
\text { Para } 0,5772 \leq y \leq 17,0
$$


BERUSKI, G. C. et al. Análise probabilística da velocidade média e caracterização da...

Sendo que:

$$
y=\ln \left(\frac{\frac{1}{N} \sum_{i=1}^{N} v_{i}}{\left(\prod_{i=1}^{N} v_{i}\right)^{\frac{1}{N}}}\right)
$$

$\beta=$ Parâmetro de escala estimado a partir de:

$$
\beta=\frac{\sum_{i=1}^{N} v_{i}}{N \alpha}
$$

$\Gamma(\alpha)=$ Função Gama ordinária de $\alpha$.

A função cumulativa de probabilidade é:

$$
F(v)=\frac{1}{\Gamma(\gamma) \beta^{\gamma}} \int_{0}^{v} v^{\gamma-1} e^{-\frac{v}{\beta}} d v
$$

A distribuição de Weibull-2 parâmetros para a velocidade do vento é expressa pela função de densidade de probabilidade:

$$
f(v)=(k / c)(v / c)^{k-1} \exp \left[-(v / c)^{k}\right]
$$

sendo a função cumulativa de probabilidade dada por:

$$
F(v)=1-\exp \left[-(v / c)^{k}\right]
$$

onde "c" é o fator de escala em unidades de velocidade do vento, "k" o fator de forma adimensional e " $v$ " a variável aleatória velocidade do vento. O fator de forma "k" está inversamente relacionado à variância $\sigma^{2}$ das velocidades eólicas em torno da média.

Os parâmetros "c" e "k" , conforme discutidos em
Justus et al. (1978), podem ser determinados a partir da transformação da equação (2) na forma linear.

$$
\ln (-\ln (1-F(v)))=-k \ln (c)+k \ln (v)
$$

que pode ser representada pela reta:

$$
Y=a+b X
$$

onde:

$$
\begin{gathered}
Y=\ln [-\ln (1-F(v)], \\
X=\ln (v), \\
a=-k \ln (c), \\
b=k .
\end{gathered}
$$

Assim, a determinação dos parâmetros "c" e "k" fica condicionada aos cálculos dos coeficientes a e $b$ da reta. Esses podem ser obtidos pelo método dos mínimos quadrados aplicado ao conjunto de dados $X=\ln (v)$ e $Y=\ln [-\ln (1-F(v))]$ obtidos dos valores de $v$ e $F(v)$ que, por sua vez, são determinados a partir das séries observadas da velocidade do vento distribuídas em $\mathrm{n}$ intervalos de classe com suas respectivas frequências.

A expressão matemática da função de densidade de probabilidade de Rayleigh é dada por:

$$
f(v)=-\frac{v}{a^{2}} \exp \left(-\frac{v^{2}}{2 a^{2}}\right)
$$

onde:

$$
a=\sqrt{\frac{2}{\pi}} E(v)
$$

$E$ (v) é a esperança matemática da variável aleatória "v" (velocidade do vento). é:

A função cumulativa de probabilidade Rayleigh

$$
F(v)=1-\exp \left(-\frac{\pi v^{2}}{4 E(v)^{2}}\right)
$$


Aplicadas as distribuições probabilísticas aos dados de velocidade média, verificou-se dentre elas qual melhor se adaptava aos dados da região em estudo. Tal avaliação foi efetuada aplicando-se o teste de Komolgorov-Smirnov aos resultados obtidos em cada distribuição.

O teste de Komolgorov-Smirnov é aplicado para verificar se os valores de uma certa amostra de dados podem ser considerados como provenientes de uma população com distribuição teórica pré-estabelecida, sob uma hipótese: a hipótese de nulidade $\left(\mathrm{H}_{0}\right)$.

$O$ teste confronta duas distribuições de frequência acumuladas, uma $F^{\prime}(X)$, teórica, e outra, $F(X)$, derivada dos dados amostrais, tal que:

- Seja $F^{\prime}(X)$ uma função de distribuição de probabilidade teórica com seus parâmetros especificados;

- Seja $F(X)$ uma distribuição de probabilidade empírica, ou seja, para uma determinada classe de frequência $F(X)=$ fa/ $N$, onde fa é a frequência acumulada da classe;

Determina-se:

$$
\text { Dmáx }=\operatorname{MAX}\left|F^{\prime}(X)-F(X)\right|
$$

Se, ao nível de significância estabelecido, o valor observado de Dmáx (calculado) for maior ou igual ao valor crítico de Dmáx (tabelado), a hipótese de nulidade, ou seja, a hipótese de que os dados amostrais provêm de uma população com distribuição teórica, $F^{\prime}(X)$ é rejeitada (ASSIS et al., 1996).

Já para a análise da direção predominante do vento, os dados foram descritos probabilisticamente através da frequência relativa simples $\left(\mathrm{fr}_{\mathrm{d}}\right)$ para cada mês do ano do período em estudo, cuja fórmula esta descrita abaixo:

$$
\mathrm{fr}_{\mathrm{d}}=\frac{\mathrm{f}_{\mathrm{d}}}{\mathrm{N}}
$$

onde $f_{d}$ é a frequência de cada direção e $N$ é o numero de observações dentro do mês analisado.

Para determinar as direções dos ventos, foram utilizados os pontos cardeais Norte, Sul, Leste e Oeste e também os pontos colaterais Nordeste, Sudoeste, Sudeste e Noroeste, através da rosa-dos-ventos.

\section{RESULTADOS E DISCUSSÃO}

Inicialmente efetuou-se a estatística descritiva dos dados referentes a uma série de 19 anos, analisando-se a velocidade média diária do vento no município da Lapa, PR (Tabela 1).

TABELA 1 - TRATAMENTO DESCRITIVO DOS DADOS DE VELOCIDADE MÉDIA DIÁRIA DO VENTO (M.S-1) PARA A LOCALIDADE DA LAPA, PARANÁ, NO PERÍODO DE 1988 A 2007

\begin{tabular}{llllllllllllll}
\hline & \multicolumn{10}{c}{ Meses } \\
\hline Estatísticas & Jan & Fev & Mar & Abr & Mai & Jun & Jul & Ago & Set & Out & Nov & Dez \\
\hline Máximo & 4,72 & 5,76 & 4,12 & 6,19 & 6,10 & 7,11 & 6,04 & 6,11 & 6,60 & 5,68 & 7,10 & 5,44 \\
Mínimo & 1,30 & 1,31 & 1,08 & 0,98 & 1,02 & 0,79 & 0,95 & 1,00 & 1,22 & 1,17 & 1,47 & 1,55 \\
Ampl. Total & 3,42 & 4,45 & 3,04 & 5,20 & 5,10 & 6,30 & 5,10 & 5,10 & 5,38 & 4,51 & 5,60 & 3,90 \\
Total de dados & 589 & 535 & 583 & 570 & 589 & 570 & 589 & 558 & 570 & 589 & 570 & 589 \\
Média Aritmética & 2,70 & 2,55 & 2,41 & 2,46 & 2,35 & 2,46 & 2,66 & 2,66 & 3,11 & 3,03 & 3,12 & 3,03 \\
D. Padrão & 0,60 & 0,61 & 0,54 & 0,66 & 0,81 & 0,93 & 0,87 & 0,89 & 0,89 & 0,80 & 0,77 & 0,73 \\
Variância & 0,37 & 0,37 & 0,29 & 0,44 & 0,66 & 0,87 & 0,76 & 0,80 & 0,79 & 0,64 & 0,60 & 0,53 \\
\hline
\end{tabular}


BERUSKI, G. C. et al. Análise probabilística da velocidade média e caracterização da...

Verificou-se que as maiores velocidades foram encontradas nos meses de junho e novembro com valores de 7,11 e $7,10 \mathrm{~m} . \mathrm{s}^{-1}$, respectivamente. As menores velocidades do vento registraram-se nos meses de Inverno com valores de 0,79 m.s. ${ }^{-1}$ para junho e 0,95 $\mathrm{m} . \mathrm{s}^{-1}$ para o mês de julho.

Como junho foi o mês que apresentou os maiores e menores valores de velocidade média do vento, verifica-se que este apresentou uma grande variação de seus registros, resultando em uma amplitude total de $6,30 \mathrm{~m} \cdot \mathrm{s}^{-1}$. Esta grande variação observada na velocidade do vento pode ser atribuída ao fato deste mês relacionar-se ao solstício de inverno. Nesta época, a região em estudo sofre com as constantes penetrações de frentes frias vindas do Sul do continente americano. Essas frentes frias alteram os campos de pressões atmosféricas, podendo influenciar diretamente na direção e na velocidade do vento (RODRIGUES et al., 2004). Com a entrada da frente fria, ocorre uma diminuição da pressão, fazendo com que o vento recue e aumente sua velocidade. Durante a sua passagem, ocorre elevação rápida da pressão atmosférica, ocasionando mudanças súbitas na direção do vento, que geralmente é acompanhado de rajadas. Já com o afastamento da frente, a pressão eleva-se lenta e continuamente, podendo apresentar rajadas com posterior estabilidade (AYOADE, 2007).

As grandes variações na velocidade média do vento na região em estudo devem-se também ao fato do município em estudo localizar-se nos Campos Gerais do Paraná. Sabe-se que o clima da região e de todo o Estado sofre influência de diversos fatores, como a infiltração das massas de ar frio, especialmente durante os meses do inverno, possivelmente a Massa Polar Atlântica; ação dos ventos marítimos úmidos influenciados pelo Anticiclone do Atlântico Sul, que ocasionam chuvas orográficas na Serra do Mar e até mesmo no interior do estado; e variações na posição do Equador Térmico e do Anticiclone do Atlântico Sul, que no verão posicionam-se ao sul e interferem na dinâmica climática do estado e dos Campos Gerais. Outro mecanismo que vem alterando o clima da região nos últimos anos são os fenômenos "El Niño" e "La Niña" (CRUZ, 2007).

Com relação à Massa Polar Atlântica, com deslocamento continental, ela origina-se sobre as águas sub-antárticas ao Sul da América do Sul, penetra no estado por Oeste e Sudoeste e predomina no outono e inverno. É seca e não adquire umidade ao longo do trajeto. A Massa Polar Atlântica com deslocamento marítimo também origina-se ao Sul do continente sul-americano, predomina durante o inverno e a primavera. É seca na origem e absorve umidade do oceano, principalmente da corrente quente do Brasil (WONS, 1982).

Estudos realizados na mesma região por Leite; Virgens Filho (2007), no município de Ponta Grossa para uma série de 26 anos de registros diários, evidenciam que as maiores oscilações de velocidades médias do vento ocorreram no período do inverno, revelando a maior amplitude total no mês de julho $\left(9,22 \mathrm{~m} \cdot \mathrm{s}^{-1}\right)$ e confirmando a interferência direta de fenômenos climáticos na região, capazes de alterar o comportamento dos ventos.

Para a região sudeste do Brasil, mais precisamente no município de Ituverava, SP, outro estudo verificou que as maiores velocidades médias do vento encontravam-se na primavera, atingindo um valor máximo no mês de setembro (MUNHOZ; GARCIA, 2008). Da mesma forma que na localidade de Ituverava, estudos realizados no Rio Grande do Sul demonstraram que na primavera verificam-se os maiores valores de velocidade média dos ventos (DA SILVA, et al., 1997; 2000; DA SILVA; BURGUEÑO, 2001).

Na sequência, aplicou-se aos dados de velocidade média do vento cinco distribuições probabilísticas: Normal, Beta, Gama, Rayleigh e Weibull, as quais foram, posteriormente, avaliadas pelo teste de KomolgorovSmirnov (K-S) a um nível de significância de $5 \%$, cujos resultados obtidos encontram-se na tabela 2 .

$\mathrm{O}$ teste K-S é utilizado para verificar quais das distribuições melhor representam os dados em estudo, de uma determinada série. Aplicado aos 19 anos de dados diários de velocidade média, verificou-se que dentre as distribuições utilizadas apenas a Beta e a Gama se ajustaram aos dados em análise, sendo que a distribuição Gama foi a que melhor se ajustou aos dados em todos os meses do período em estudo, representando de forma satisfatória os dados de velocidade média dos ventos no município da Lapa, PR.

Tendo em vista sua grande importância, vários estudos probabilísticos têm sido realizados com o objetivo de caracterizar o vento em várias regiões, porém são poucos os registros que evidenciam a utilização da distribuição Gama para caracterizar o vento. Dentre as distribuições de probabilidade, a Beta e a Weibull com suas variações são as mais comumente utilizadas em trabalhos de caracterização eólica das regiões estudadas. Análises como as realizadas em Ponta Grossa, PR, por Leite e Virgens Filho (2006), nas quais se aplicou aos dados de velocidade média dos ventos as distribuições Beta, Rayleigh e Weibull, verificou-se que a distribuição Beta foi a que melhor representou os dados de velocidade média na área em estudo. Assim como para o município de Ponta Grossa, estudos efetuados para outras localidades do Brasil também apontam a 
BERUSKI, G. C. et al. Análise probabilística da velocidade média e caracterização da...

distribuição Beta como uma boa alternativa para o ajuste de séries de dados de velocidade média do vento, como é o caso de Botucatu, SP (MARQUES JUNIOR et al., 1995), Passo do Lontra/Pantanal Sul-Mato-Grossense (SOUZA et al., 1998) e Panorama, SP (SANSIGOLO, 2005). Conforme citado anteriormente, a distribuição probabilística de Weibull também é amplamente uti- lizada para se determinar características eólicas de diversas regiões. Estudos aplicados à região Nordeste do Brasil, onde se analisaram as características eólicas regionais e um possível potencial para a implementação de torres de captação de energia, verificaram que esta distribuição representou adequadamente os dados em análise (DA SILVA et al., 2002; 2004).

TABELA 2 - VALORES DO TESTE DE KOMOLGOROV-SMIRNOV (5\%) APLICADOS A SÉRIE HISTÓRICA DE DADOS DE VELOCIDADE

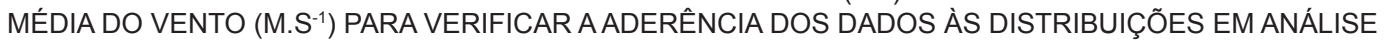

\begin{tabular}{lcccccc}
\hline & & NORMAL & BETA & GAMA & WEIBULL & RAYLEIGH \\
\cline { 2 - 6 } & Dmáx5\% & Dcalc & Dcalc & Dcalc & Dcalc & Dcalc \\
\hline Janeiro & 0,0560 & 0,0294 & 0,0232 & 0,0130 & 0,1040 & 0,2264 \\
Fevereiro & 0,0588 & 0,0508 & 0,0302 & 0,0231 & 0,1426 & 0,2377 \\
Março & 0,0563 & 0,0482 & 0,0347 & 0,0341 & 0,1418 & 0,2492 \\
Abril & 0,0570 & 0,0468 & 0,0298 & 0,0176 & 0,1641 & 0,2035 \\
Maio & 0,0560 & 0,0625 & 0,0368 & 0,0240 & 0,0965 & 0,1435 \\
Junho & 0,0570 & 0,0732 & 0,0485 & 0,0311 & 0,1230 & 0,1369 \\
Julho & 0,0560 & 0,0437 & 0,0324 & 0,0145 & 0,0772 & 0,1471 \\
Agosto & 0,0576 & 0,0395 & 0,0270 & 0,0179 & 0,0778 & 0,1398 \\
Setembro & 0,0570 & 0,0308 & 0,0265 & 0,0115 & 0,1274 & 0,1922 \\
Outubro & 0,0560 & 0,0305 & 0,0327 & 0,0154 & 0,0774 & 0,1869 \\
Novembro & 0,0570 & 0,0269 & 0,0149 & 0,0078 & 0,1212 & 0,2166 \\
Dezembro & 0,0560 & 0,0216 & 0,0232 & 0,0166 & 0,0703 & 0,2317 \\
\hline
\end{tabular}

Tais resultados comprovam a necessidade de se efetuar estudos regionalizados testando-se um maior número de modelos probabilísticos para o ajuste desta variável climática, uma vez que peculiaridades do espaço físico e a interferência de fenômenos climáticos na região, em escala mensal, diária e até mesmo horária, são capazes de alterar o comportamento da variável climática vento de forma significativa. Conforme mostrado na Tabela 2 e discutido anteriormente, a distribuição Beta também ajustou adequadamente os dados de velocidade média do vento no município da Lapa, no entanto, a distribuição Gama revelou ajustes melhores em todos os meses, evidenciando que esta última representa melhor os dados desta região. Este resultado extremamente importante só pôde ser verificado pela investigação de um modelo probabilístico menos utilizado em outros estudos e regiões.

Com relação à direção predominante do vento, os resultados da análise encontram-se na Tabela 3.

As análises realizadas com dados diários de direção predominante do vento no município da Lapa, PR demonstraram que as direções Leste, Nordeste e Norte predominaram ao longo do ano, tendo estas, respectivamente, os seguintes valores médios $33,42 \%$, 30,86 e $15,77 \%$. Analisando-se as direções mês a mês verificou-se que entre os meses de outubro a abril predominou a direção Leste, enquanto de maio a setembro, a direção predominante foi a Nordeste. $O$ fato de a região apresentar ventos predominantes nas direções Leste e Nordeste deve-se provavelmente aos centros de alta pressão do Atlântico que geram ventos nessas direções (TUBELIS; NASCIMENTO, 1986). 
BERUSKI, G. C. et al. Análise probabilística da velocidade média e caracterização da...

TABELA 3 - FREQUÊNCIA RELATIVA PARA OS DADOS DE DIREÇÃO PREDOMINANTE, PARA A LOCALIDADE DA LAPA, PR, NO PERÍODO DE SETEMBRO DE 1988 A JULHO DE 2007

\begin{tabular}{|c|c|c|c|c|c|c|c|c|}
\hline \multirow[b]{2}{*}{ Meses } & \multicolumn{8}{|c|}{ Direções } \\
\hline & $\underline{N}$ & $\underline{\mathrm{NE}}$ & $\underline{E}$ & $\underline{\mathrm{SE}}$ & $\underline{S}$ & $\underline{\mathrm{SW}}$ & $\underline{W}$ & NW \\
\hline Janeiro & 10,1737 & 20,8437 & 42,9280 & 4,2184 & 0,7444 & 2,4814 & 6,4516 & 12,1588 \\
\hline Fevereiro & 16,0079 & 24,5059 & 37,1542 & 3,7549 & 1,9763 & 2,3715 & 6,9170 & 7,3123 \\
\hline Março & 14,0652 & 25,0429 & 44,5969 & 4,4597 & 1,0292 & 1,5437 & 4,9743 & 4,2882 \\
\hline Abril & 13,6842 & 31,5789 & 34,3860 & 2,6316 & 2,6316 & 4,2105 & 6,6667 & 4,2105 \\
\hline Maio & 15,7706 & 32,7957 & 27,5986 & 2,8674 & 4,4803 & 6,8100 & 5,3763 & 4,3011 \\
\hline Junho & 26,8519 & 38,3333 & 12,2222 & 2,5926 & 2,7778 & 6,4815 & 5,3704 & 5,3704 \\
\hline Julho & 25,9857 & 33,3333 & 18,8172 & 1,0753 & 3,0466 & 7,7061 & 5,0179 & 5,0179 \\
\hline Agosto & 20,8729 & 40,9867 & 21,6319 & 2,6565 & 2,6565 & 4,7438 & 3,2258 & 3,2258 \\
\hline Setembro & 13,1206 & 37,7660 & 33,5106 & 1,9504 & 2,8369 & 3,7234 & 2,8369 & 4,2553 \\
\hline Outubro & 10,6830 & 33,0998 & 40,6305 & 1,2259 & 1,9264 & 4,0280 & 4,0280 & 4,3783 \\
\hline Novembro & 10,0000 & 25,4386 & 46,1404 & 2,4561 & 1,9298 & 3,5088 & 3,8596 & 6,6667 \\
\hline Dezembro & 12,0543 & 26,6553 & 41,4261 & 2,7165 & 1,5280 & 2,3769 & 7,1307 & 6,1121 \\
\hline $\begin{array}{l}\text { Total de } \\
\text { ocorrências }\end{array}$ & 189,2699 & 370,3802 & 401,0425 & 32,6052 & 27,5638 & 49,9857 & 61,8553 & 67,2972 \\
\hline $\begin{array}{l}\text { Média de } \\
\text { ocorrências }\end{array}$ & 15,7725 & 30,8650 & 33,4202 & 2,7171 & 2,2970 & 4,1655 & 5,1546 & 5,6081 \\
\hline
\end{tabular}

No período estudado, verificou-se que no mês de maio ocorre uma inversão da direção, passando de Leste para Nordeste, fato este que se mantém até o mês de outubro, quando a direção predominante volta a ser a Leste. Essa mudança na direção do vento na região deve-se à presença no continente de uma massa de ar estacionária que pode se aquecer, tornando esta mais amena e permitindo a entrada de outras massas no continente (CRUZ, 2007).

A seguir encontra-se a representação gráfica (Figura 1) dos dados diários de direção predominante, para a localidade em estudo, demonstrando o comportamento do vento na região.

Leite e Virgens Filho (2006), em estudos objetivando caracterizar os ventos da localidade de Ponta Grossa, PR, verificaram que os mesmos se deslocavam com maior frequência na direção Nordeste, com valores médios de $49,10 \%$, tendo como segunda e terceira direções predominantes Noroeste e Leste, respectivamente. Já para Maringá, município localizado no Norte do Paraná, Carfan et al. (2007) evidenciaram que os ventos predominam na direção Nordeste. Tais resultados corroboram o comportamento da direção do vento observado neste estudo, evidenciando, no Estado do Paraná, influências diretas de vários fatores, como infiltração de massas de ar frio, ação de ventos úmidos e variações do Equador Térmico e do Anticiclone do Atlântico Sul.

Da Silva et al. (1997) verificaram que para a região de Pelotas, RS, os ventos na primavera e no verão se mantiveram na direção Leste; no outono, prevaleceu a direção Sudoeste e no inverno, a Nordeste, evidenciando que tal região apresenta ventos com comportamentos semelhantes à região da Lapa.

Em estudos realizados no Sudeste brasileiro, Munhoz e Garcia (2008) verificaram na região de Ituverava, $\mathrm{SP}$ que os ventos predominaram durante a maior parte do ano no sentido Sudeste, apresentando somente nos meses de verão a direção predominante Noroeste. Ainda na mesma região brasileira na localidade de Panorama, $\mathrm{SP}$, verificou-se que os ventos predominavam na direção Sudeste, na maior parte do ano, sendo seguidos por Leste e Nordeste (SANSIGOLO, 2005).

Para o Nordeste do Brasil, verificou-se que as correntes de ar variam de Norte a Sul, entretanto, ao longo do ano verifica-se que a direção predominante dos ventos na região é a Leste, seguida pela Sudeste e em menor frequência a Nordeste (DA SILVA et al., 2002; 2004). 
BERUSKI, G. C. et al. Análise probabilística da velocidade média e caracterização da...

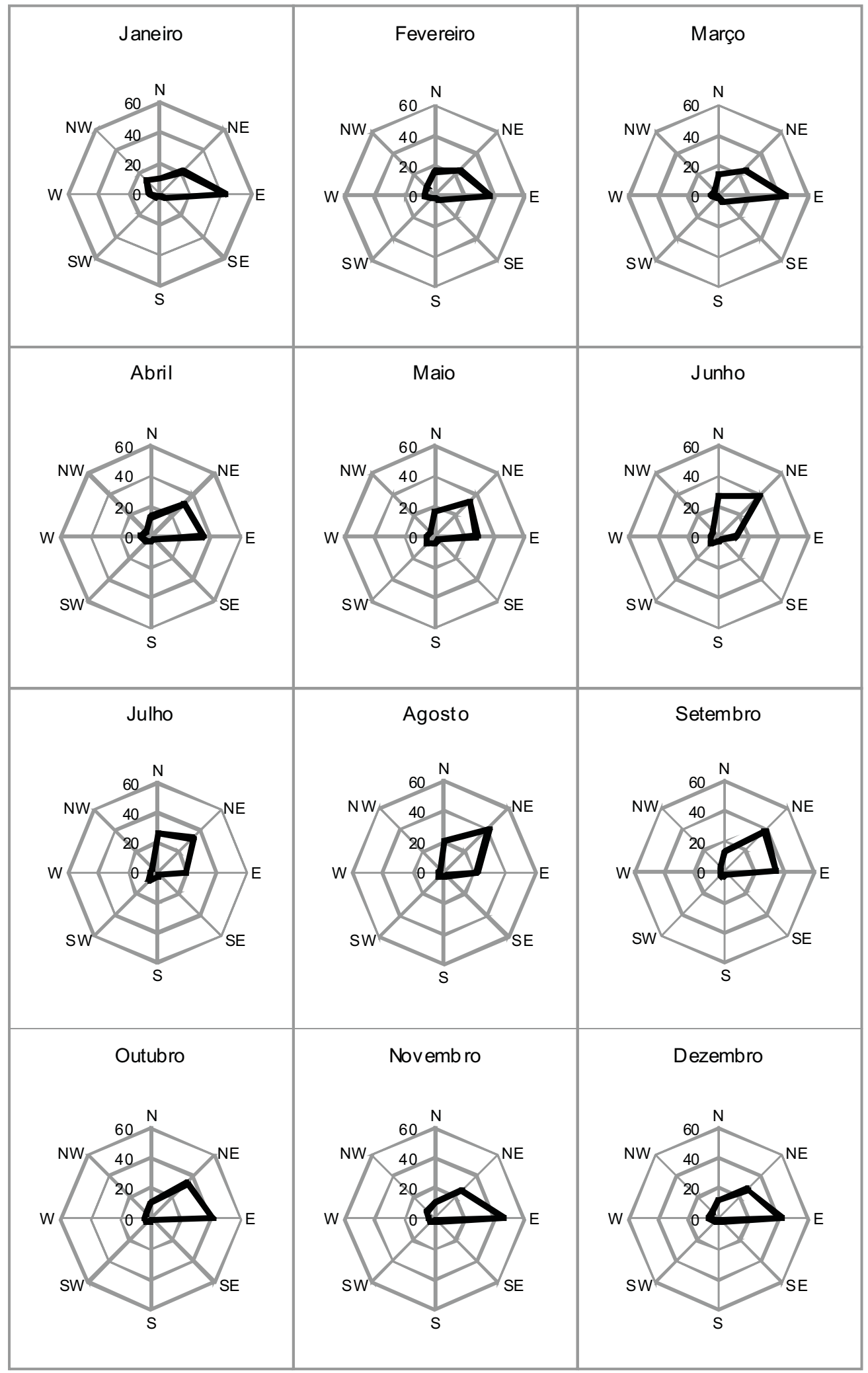

FIGURA 1 - DIREÇÃO MÉDIA MENSAL PREDOMINANTE DO VENTO (\%) NA LAPA, PR - 1988 A 2007 
BERUSKI, G. C. et al. Análise probabilística da velocidade média e caracterização da...

\section{CONCLUSÃO}

O mês de junho foi o que apresentou o valor máximo de velocidade do vento $\left(7,11 \mathrm{~m} . \mathrm{s}^{-1}\right)$ e o menor valor $\left(0,79 \mathrm{~m} . \mathrm{s}^{-1}\right)$, resultando numa grande amplitude $\left(6,30 \mathrm{~m} . \mathrm{s}^{-1}\right)$, sendo esta variabilidade, provavelmente, decorrente das entradas constantes de frentes frias vindas de região Sul do continente.

A Distribuição Gama foi a que melhor se ajustou aos dados de velocidade média do vento podendo esta ser considerada um ótimo modelo para representar os dados da região em que se efetuou a pesquisa.

A direção predominante do vento ao longo do ano foi a Leste $(33,42 \%)$, sendo seguida pela Nordeste $(30,86 \%)$ e Norte $(15,77 \%)$.

\section{AGRADECIMENTOS}

Ao Instituto Agronômico do Paraná (IAPAR), área de Ecofisiologia, pela cessão dos dados, o qual possibilitou a realização do presente estudo. A Fundação Araucária pelo suporte financeiro ao projeto de pesquisa, permitindo assim o seu desenvolvimento e ao Conselho Nacional de Desenvolvimento Científico e Tecnológico (CNPq) pela bolsa de iniciação cientifica.

\section{REFERÊNCIAS}

ALDABÓ, R. Energia Eólica. São Paulo: Artliber Editora, 2002. $160 \mathrm{p}$.

ASSIS, F. N.; ARRUDA, H. V.; PEREIRA, A. R. Aplicações de estatística à climatologia. Pelotas: Ed. Universitária / UFPel, 1996. $161 \mathrm{p}$.

AYOADE, J. O. Introdução à climatologia para os trópicos. 12. ed. Rio de Janeiro: Ed. Bertrand Brasil, 2007. 332 p.

CARFAN, A.C.; NERY, J.T.; STIVARI, S.M. Dinâmica dos ventos e temperatura do ar em Maringá, no Verão de 2004. Revista Eletrônica Geografar, Curitiba, v. 2, n.1, p. 01-21, jan./jun. 2007.

CRUZ, G.C.F. Alguns aspectos do clima nos Campos Gerais. In: MELO, M.S.; MORO, R.S.; GUIMARÃES, G.B. (Orgs.). Patrimônio Natural dos Campos Gerais do Paraná. Ponta Grossa: Editora UEPG, 2007. Capítulo 5, p. 59-72.
DA SILVA, B.B.; ALVES, J. J. A.; CAVALCANTI, E.P.; DANTAS, R.T. Potencial eólico na direção predominante do vento no Nordeste brasileiro. Revista Brasileira de Engenharia Agrícola e Ambiental, Campina Grande, v. 6, n. 3, p. 431-439, 2002.

DASILVA, B.B.; ALVES, J.J.A.; CAVALCANTI, E.P.; VENTURA, E.D. Variabilidade espacial e temporal do potencial eólico da direção predominante do vento no Nordeste do Brasil. Revista Brasileira de Meteorologia, v.19, n. 2, p.189-202, 2004.

DA SILVA, J.B.; BURGUEÑO, L.E.T. Estimativa do potencial eólico a partir de tabelas de probabilidades da velocidade média pentadal do vento, na Cascata, em Pelotas - RS. Revista Brasileira de Agrometeorologia, Santa Maria, v. 9, n. 2, p. 333-338, 2001.

DA SILVA, J.B.; LARROZA, E.G.; BURGUEÑO, L.E.T. Estimativa do potencial eólico no Campus da Universidade Federal de Pelotas, em Pelotas, RS. Revista Brasileira de Agrometeorologia, Santa Maria, v. 8, n.1, p.111-119, 2000.

DASILVA, J.B.; SCHONS, R.L.; LARROZA, E.G. Probabilidade de ocorrência de rajadas máximas de vento em Pelotas, RS. Revista Brasileira de Agrometeorologia, Santa Maria, v. 5, n. 2, p. 237-240, 1997.

FALLS, L. W. The beta distribution: a statistical model for world cloud cover. Alabama, NASA, 1973, p.1-6. (NASA Tecnical Memorandum, TMX-64714).

GREENWOOD, J. A.; DURAND, D. Aids for fitting the gamma distribution by maximum likelyhood. Technometrics, v. 2, n.1, p. 55-65, 1960.

JUSTUS, C. G.; HARGRAVES, W.R.; MIKHAIL, A.; GRABER, D. Methods for estimating wind speed frequency distributions. J. Appl. Meteor., v.17, n. 3, p. 350-353, 1978.

LEITE, M.L.; FILHO, J.S.V. Avaliação da distribuição Beta com modelo probabilístico para análise de dados de velocidade do vento para Ponta Grossa - PR. Publicatio UEPG, Ciências Exatas e da Terra, Ciências Agrárias e Engenharias, Ponta Grossa, v. 23, n.1, p. 51-57, abril, 2007.

LEITE, M.L.; FILHO, J.S.V. Avaliação da velocidade média e direção predominante do vento em Ponta Grossa - PR. Revista Brasileira de Agrometeorologia, Santa Maria, v.14, n. 2, p. 157-167, 2006.

MARQUES JUNIOR, S.; VALADÃO, L.T.; VIEIRA, A.R.R.; MOURA, M.V.T. Análise de dados de vento para a região de Botucatu - SP utilizando a distribuição Beta. Revista Brasileira de Agrometeorologia, Santa Maria, v. 3, p.129-132, 1995.

MUNHOZ, F.C.; GARCIA, A. Caracterização da velocidade e direção predominante dos ventos para a localidade de Ituverava-SP. Revista Brasileira de Meteorologia, v. 23, n.1, p.30-34, 2008.

OLIVEIRA, C.E.L.; FRÜHLING, I.; URIBE-OPAZO, M.A. Análise climatológica do potencial eólico na região de Cascavel - PR. Engenharia Agrícola. Jaboticabal, v. 23, n. 3, p. 425433, set/dez. 2003. 
PEARSON, K. Tables of the incomplete Beta function. London: University College, Biometriks Office, 1934. 494p.

RODRIGUES, M. L.; FRANCO, D.; SUGAHARA, S., Climatologia de frentes frias na litoral de Santa Catarina. Revista Brasileira de Geofísica, v. 22, n. 2, p. 135-151, 2004.

SANSIGOLO, C.A. Distribuição de probabilidade de velocidade e potência do vento. Revista Brasileira de Meteorologia, v. 20, n. 2, p. 207-214, 2005.
SOUZA, A.; SOLER, L.S.; FERNANDES, W.A. Probabilidade da velocidade média mensal do vento no Passo do Lontra/ Pantanal Sul-Mato-Grossense: um estudo preliminar. Revista Brasileira de Agrometeorologia, Santa Maria, v. 6, n. 2, p. 235-238, 1998.

TUBELIS, A.; NASCIMENTO, F.C.L. Meteorologia descritiva - Fundamentos e aplicações brasileiras. 1. ed. São Paulo: Nobel, 1986. 374 p.

WONS, I. Geografia do Paraná com fundamentos de Geografia geral. 4. ed. Curitiba: Ed. Ensino Renovado, 1982. 172 p. 